Ayşegül Dere
Fırat University, a.deredfirat.edu.tr, Elazığ-Turkey

DOI $\quad$ http://dx.doi.org/10.12739/NWSA.2018.13.4.3A0087

ORCID ID $0000-0003-1744-1315$

CORRESPONDING AUTHOR Ayşegül Dere

\title{
GÜNEŞ TAKİP SİSTEMLERI İÇİN Al/P-Si/Zn:CuO/Al FOTODİYOTLARI
}

Öz

Bu çalışmada, Zn katklı CuO ince filmleri hazırlanarak Al-p-Si/Zn katkılı CuO/Al fotodiyotları üretilmiştir. Üretilen fotodiyotların akım-voltaj (I-V) ölçümleri karanlıkta ve farklı aydınlatma şiddetleri altında alınmıştır. Al-p-Si/Zn katkılı CuO/Al fotodiyotları iyi bir doğrultma ve iyi bir fototepki özelliği göstermiştir. 0.1 Zn katkılı

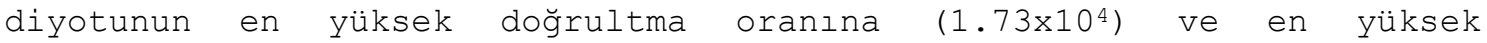
fototepki'ye $\left(2.07 \times 10^{3}\right)$ sahip olduğu saptanmıştır. Frekansa bağlı kapasite-voltaj ölçümleri oda sıcaklığında $10 \mathrm{kHz}$ ile $1 \mathrm{MHz}$ aralığında yapılmıştır. Diyotların kapasitansının, artan frekans ile azalması, arayüz yük dağılımından kaynaklanmaktadır. Anlık fotoakım ölçümleri, diyotların fotoiletkenlik davranış sergilediğini gösterir. Zn katkılı CuO filmlerin optiksel özellikleri de UV-VIS spektroskopisi ile incelenmiş ve optik enerji bant aralığı 1.89-2.15 eV aralığında bulunmuştur. Filmlerin morfolojik yüzey özellikleri atomik güç mikroskobu (AFM) ile araştırılmıştır. Filmlerin yüzey morfolojisi Zn katkısına bağlı olarak değişmiştir. Elde edilen sonuçlar, Al/pSi/Zn:CuO/Al diyotlarının güneş izleme sistemlerinde fotosensör olarak kullanılabileceğini göstermiştir.

Anahtar Kelimeler: Heteroeklem Diyot, Zno İnce Filmler, Güneş Takip Sistemi, Al, CuO

\section{Al/P-Si/Zn:CuO/Al PHOTODIODES FOR SOLAR TRACKING SYSTEMS}

\section{ABSTRACT}

In this study, Zn-doped CuO thin films were produced to prepare Al-p-Si/Zn-doped CuO/Al photodiodes. The current-voltage (I-V) measurements of the photodiodes produced are taken in the dark and under different illumination intensities. Al-p-Si/Zn-doped CuO/Al photodiodes showed good rectification and good photoresponse properties. ㅇ.1 $\mathrm{Zn}$ doped diodes have the highest rectification ratio $\left(1.73 \times 10^{4}\right)$ and the highest photoresponse $\left(2.07 \times 10^{3}\right)$. Frequencydependent capacity-voltage measurements were analyzed at room temperature between $10 \mathrm{kHz}$ and $1 \mathrm{MHz}$. The capacitance of the diodes decreases with increasing frequency, the load distribution is due to the interface. Transient photocurrent measurements indicate that the diodes exhibit photoconductivity behavior. The optical properties of Zn-doped CuO films were investigated by UV-VIS spectroscopy and the optical energy band gap was found at $1.89-2.15 \mathrm{eV}$. The morphological surface properties of the films were investigated by atomic force microscopy (AFM). The surface morphology of the films varied depending on the $\mathrm{Zn}$ doped. The results obtained have shown that Al/p$\mathrm{Si} / \mathrm{Zn}: \mathrm{CuO} / \mathrm{Al}$ diodes can be used as photosensors in solar tracking systems.

Keywords: Heterojuntion Diode, ZnO Thin Films, Solar Tracking Systems, Al, CuO

How to Cite:

Dere, A., (2018). Güneş Takip Sistemleri İçin Al/P-Si/Zn:Cuo/Al Fotodiyotları, Physical Sciences (NWSAPS), 13(4):64-75, DOI: 10.12739/NWSA.2018.13.4.3A0087. 


\section{GİRIŞ (INTRODUCTION)}

Son yıllarda, fotovoltaik uygulamalarda yeni gelişmiş ve fonksiyonel materyallere ihtiyaç vardır [1]. Metal oksitler, Sitabanlı mikroelektronik özelliği, düşük maliyeti ve yüksek uyumluluklarından dolayı büyük ilgi görmüştür. Metal oksitler sadece bilimsel olarak değil, aynı zamanda gaz algılama, elektronik ve fotonik cihazlardaki potansiyel uygulamalar için de caziptir [2]. Bu metal oksitler arasında olan CuO ilginç bir metal oksittir [2 ve 3]. (CuO) dar bant aralıklı, yarı iletken bir metal oksittir. 1.2-1.9 eV [4] Cuo'nun dar bir bant aralığına sahip olması, spektrumun görünür bölgesinde yüksek absorbasyon sağlamaktadır. Dolayısıyla heteroeklemli güneş pillerinde, elektronikte ve sensör uygulamalarında kullanılmaktadır [5]. Bunun dışında gaz sensörü olarak da kullanımı oldukça yaygındır. Bakır (II) oksit (CuO), zehirli olmayan, doğada bol miktarda bulunabilen, üretim maliyeti düşük, kahverengi veya siyaha yakın oldukça koyu bir renge sahip parlak bir katıdır ve p-tipi bir yarıiletken malzemedir [6, 7, 8 ve 9]. Görünür bölgedeki yüksek emilim katsayısı, toksik olmaması ve ucuz üretimi nedeniyle önemli mineral oksit materyallerinden biridir [10]. CuO ince filmler güneş hücreleri [11], manyetik depolama ortamları, kataliz [12], gaz sensörleri [13 ve 15] ve lityum hücrelerde elektrotlar için uygundur [16]. Diğer bir metal oksit olan çinko oksit ( $\mathrm{ZnO})$, geniş bant aralığına ( 3.37 eV) sahip, kendiliğinden n-tipi metal oksit yarı iletkendir. Optoelektronik cihazlar ve gaz sensörleri için yaygın olarak incelenmiştir [17 ve 18]. Bu p ve n-tipi yarı-iletken tabakaların, yani cuo/Zno yapısının ortak bir şekilde üretilmesiyle, bir p-n heteroeklemi oluşturulabilir. Son zamanlarda CuO-Zno bileşikler ve CuO/Zno heteroeklemler üzerinde yapılan çalışmalar bu yapıların verimli özellliklere sahip olduklarını göstermiştir [19].

Literatüre göre bu yarıiletken metal-oksit heteroeklem yapılar, iki sinterlenmiş metal katmanın mekanik olarak preslenmesi [20], RF Magnetron püskürtme [21], sol-jel [22] ve elektron-depolama gibi çeşitli yollarla üretilir [23]. $\mathrm{H}_{2}, \mathrm{NO}_{2}, \mathrm{CO}, \mathrm{H}_{2} \mathrm{~S}$ gaz sensörleri, güneş pilleri, güneş enerjisi üretimi ve fitokataliz gibi çeşitli uygulamalar için kullanılırlar [20 ve 25]. Fotovoltaik uygulamalar için kullanılan malzemelerin yanı sıra panel yani altlık malzemesi de oldukça önemlidir. Son 30 yıldır yaygın olarak kullanılan fotovoltaik panel malzemesi o24 verimi aşan silikondur (Si). Silikonun pahalı olması nedeniyle ince film teknolojisiyle oluşan paneller gelişme göstermektedir. Dünyada, fotovoltaik panel üretimi ortalama olarak yıllık \%30 civarında bir artış göstermektedir. Değişen iklim şartları ve güneş ışığı eksikliğinden dolayı paneller ekonomik olmayabilir. Bu yüzden panellerin ışığı absorbe etme ve algılama özelliği çok iyi olmalıdır Herhangi bir fotovoltaik sisteminin çıkış gücü, sisteme giren güneş enerjisine bağlıdır. Sisteme daha fazla güneş enerjisi girişi sağlamak için güneşi takip etmesi gerekmektedir. Fotovoltaik panel üzerine yapılan tüm çalışmaların ana fikri yatırım geri dönüş süresini azaltmaya yöneliktir. Bu da ancak üretilen panelin verimliliğini artırarak gerçekleştirilebilir. Üretilen panelin verimliliğinin arttırılması da ancak panelin üzerine düşen ışınım şiddetinin arttırılması yani uygun kaplama malzemesi sayesinde gerçekleştirilir [26].

\section{2. ÇALIŞMANIN ÖNEMI (RESEARCH SIGNIFICANCE)}

Bu çalışmada Zn katkılı CuO filmler, basit, düşük maliyetli uygun bir yöntem olan sol-jel spin kaplama yöntemiyle hazırlanmış, bunların $\mathrm{Al} / \mathrm{p}-\mathrm{Si} / \mathrm{Zn}-\mathrm{CuO} / \mathrm{Al}$ yapılı heteroeklem fotodiyotları üretilmiştir. Fotodiyotun elektrik, optik ve fotoduyarlılık özellikleri araştırılmıştır. Fotodiyodun fotoiletkenlik özellikleri, ışığa bağlı 
akım voltaj ve anlık foto akım ölçümleri ile incelenmiştir. Ayrıca, elektriksel özellikleri, frekansa bağlı kapasite -voltaj ölçümleri ile analiz edilmiştir.

\section{DENEYSEL ÇALIŞMA (EXPERIMENTAL METHOD-PROCESS)}

0.5 molar bakır (II) asetat $\left(\left(\mathrm{CH}_{3} \mathrm{COO}\right)_{2} \cdot \mathrm{nH}_{2} \mathrm{O}\right)$ başlangıç malzemesi ve çinko asetat dehidrat $\left(\mathrm{Zn}\left(\mathrm{CH}_{3} \mathrm{COO}\right)_{2} .2 \mathrm{H}_{2} \mathrm{O}\right)$ katkı maddesi olarak kullanılmıştır. Çözücü olarak $10 \mathrm{ml}$ 2-metoxyethanol, ve stabilizatör olarak monoetanolamin kullanılmıştır. Sol-jel yöntemiyle saf cuO ve \%0.1, 0.5, 2 atomik oranlarında Zn katkılı CuO çözeltileri, manyetik karıştırıcı da $60^{\circ} \mathrm{C}^{\prime} \mathrm{de} 400 \mathrm{rpm}$ de 1 saat karıştırılmıştır. Diyot yapımında kullanılacak altıı̆ın organik ve mekanik kirlerden arındırılması oldukça önemlidir. p-tipi silisyum altıı̆̆ı kimyasal olarak, basit RCA yöntemiyle 5dk asetonda, $5 \mathrm{dk}$ deiyonize su da, $5 \mathrm{dk}$ etil alkolde ve 5dk deiyonize su da ultrasonik olarak yıkanmıştır. Daha sonra altlık, $\mathrm{HF}: \mathrm{H}_{2} \mathrm{O}$ (1:10ml) çözeltisine 30sn daldırılarak ince silikon dioksit tabakası ve ağır metaller kaldırıldıktan sonra deiyonize su ile iyice yıkanarak azot gazı ile kurutulup, alüminyum omik kontak yapımı için nanovak termal buharlaştırma cihazına yerleştirilmiştir. Buharlaştırma sisteminin vakum değeri yaklaşık 5x10torr değerine düşünce 99.99 saflıktaki alüminyum metali buharlaştırılmıştır. p-tipi silisyuma, alüminyum buharlaştırıldıktan sonra $570^{\circ} \mathrm{C}^{\prime} \mathrm{de}, 5 \mathrm{dk}$ tavlanarak omik kontak işlemi tamamlanmıştır. Bu işlemden sonra, Al-p-tipi silisyum altlıklar uygun boyutta kesilip, $5 \mathrm{dk}$ deiyonize suda ultrasonik banyo da yıkanıp azot gazı ile kurutulmuştur. Ayrıca Zn:CuO filmlerini mikroskop camı üzerine kaplamak için, camlar sırasıyla aseton, metil alkol ve deiyonize su ile 5 dakika yıkanmış ve kurutulmuştur. Hazırlanan Zn:CuO çözeltileri, mikroskop camı ve p-tipi silisyum altlıklar üzerine spin kaplama yöntemiyle $1000 \mathrm{rpm}$ de $30 \mathrm{sn}$ boyunca kaplanmıştır. Daha sonra filmler $150^{\circ} \mathrm{C}$ de hot plate üzerinde kurutulup $400^{\circ} \mathrm{C}^{\prime}$ de tavlanmıştır. Kaplama işlemi uygun kalınlığı bulmak için 5 kez tekrarlanmıştır. Film kaplama sürecinden sonra, diyotların üst Al kontağı yapılmıştır. Sonuçta, Al/p-Si/Zn:CuO/Al heteroeklem diyotları elde edilmiştir. Hazırlanan ince filmlerin optik karakterizasyonu, Shimadzu UV-VIS-NIR 3600 spekrometresi ve yüzey morfolojileri Park sistem atomik kuvvet mikroskobu (AFM) yardımıyla incelenmiştir. Diyotların elektriksel karakterizasyonu FYTRONIX-FY-7000 elektriksel karakterizasyon cihazı ile yapılmıştır.

\section{BULGULAR VE/VEYA TARTIŞMALAR (FINDINGS AND DISCUSSIONS \\ 4.1. Zn Katkılı CuO Ince Filmlerin Optik ve Yüzey Morfoloji Özellikleri (Optical and Surface Morphology Properties of Zn Doped Cuo Thin Films)}

Zn katkılı ve katkısız CuO ince filmlerin optik özellikleri UVVIS-NIR 3600 spekrometresi kullanılarak alınmıştır. 200-1000nm dalga boyu aralığındaki soğurma (absorbsiyon) spektrumları Şekil $1^{\prime}$ de verilmiştir. Şekilde görüldüğü gibi yüksek soğurma, o0.1 Zn katkılı CuO filmde yaklaşık $280 \mathrm{~nm}$ dalga boyunda görülmüştür. Soğurma spektrumundaki ani artış, temel soğurma sınırına karşılık gelmektedir. Zn katkılı CuO ince filmlerin optik band aralığı, soğurma spektrum verilerinden yararlanarak Tauc ifadesi ile analiz edilir. Optik soğurma katsayısı ( $\alpha$ ) aşağıdaki denklem ile hesaplandı [27 ve 28];

$$
\alpha=2.303 \mathrm{~A} / \mathrm{d}
$$

Burada d film kalınlığı (12.8nm), A optik soğurma ve o ise optik soğurma katsayısıdır. Üretilen filmlerin optik banda aralıkları [27 ve 28];

$$
\alpha h v=B\left(h v-E_{g}\right)^{n}
$$


İfadesi ile hesaplanır. İfade de $B$ değeri bir sabit $E_{g}$ optik bant aralığı, $n$ ise optik soğurma sürecini belirleyen bir sabittir [29]. Dolaylı ve doğrudan izinli geçişler için, $n$ değeri sırasıyla 2 ve $1 / 2$ değerlerini alır. CuO ve Zn-katkılı CuO filmleri için ( $\alpha h v)^{2 \prime}$ nin hv'ye karşı grafiği Şekil 2'de gösterilmiştir. Filmlerin optik bant aralığı Tablo 1'de verilmiştir. Ince filmlerin optik bant aralığı değerleri Zn katkısı ile değişmiştir.

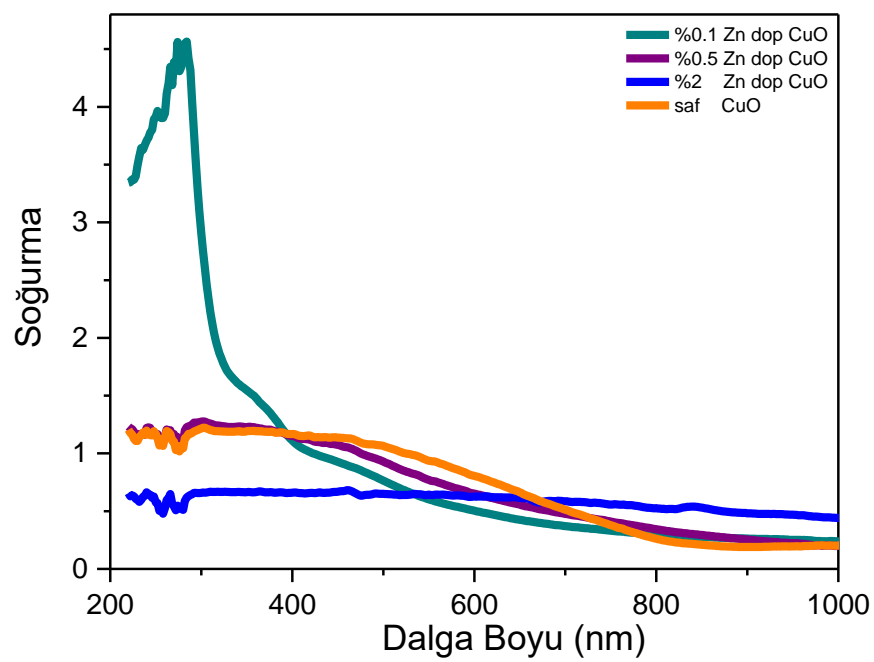

Şekil 1. Zn katkılı CuO ince filmlerin ve katkısız CuO'nun soğurma spektrumu

(Fiqure 1. Absorption spectra of Zn doped CuO thin films and pure CuO)

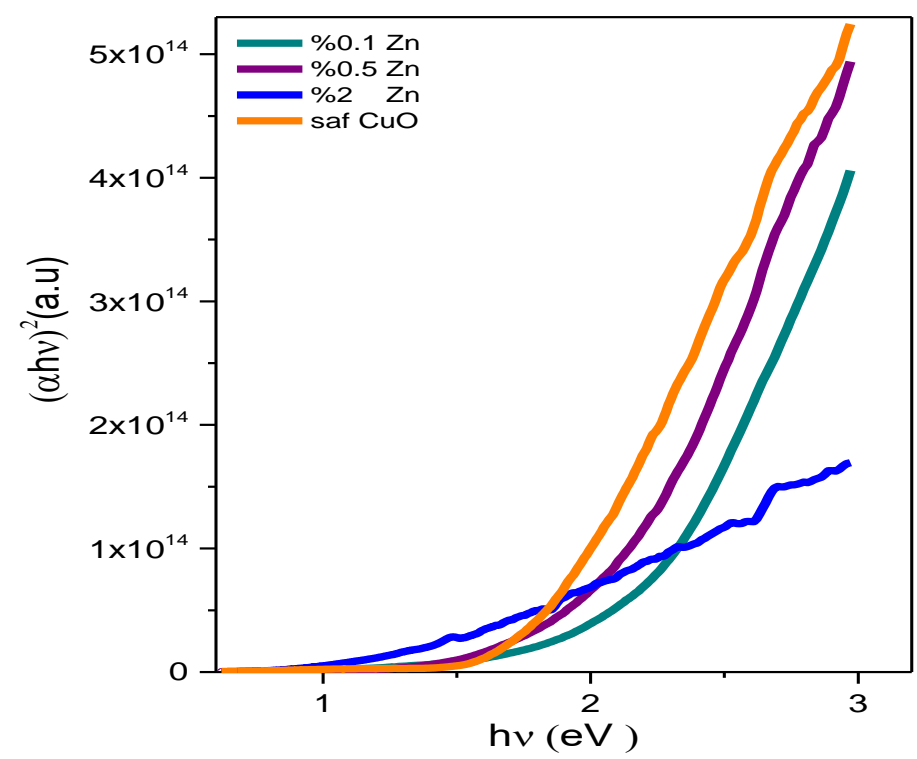

Şekil 2. Zn katkılı CuO ince filmlerin ve katkısız CuO'nun optik bant aralığı (Eg) spektrumu

(Fiqure 2. Optical bandgap (Eg) spectra of Zn doped CuO thin films and pure $\mathrm{CuO}$ )

Zn katkılı CuO ince filmlerin ve saf CuO nun yüzey morfolojisi Park Sistem XEI Atomik kuvvet mikroskobu ile analiz edilmiştir. İnce filmlerin yüzey yapıları Şekil 3, 4, 5, 6 ve 7'de gösterilmiştir. Filmlerin yüzey pürüzlülüğü, tane boyutu gibi yüzey parametreleri Tablo 1'de verilmiştir. Tablo 1'de gösterildiği gibi katkılama 
Dere, A.,

miktarına bağlı olarak yüzey pürüzlülüğü ve tane boyutunda artış gözlenmiştir.
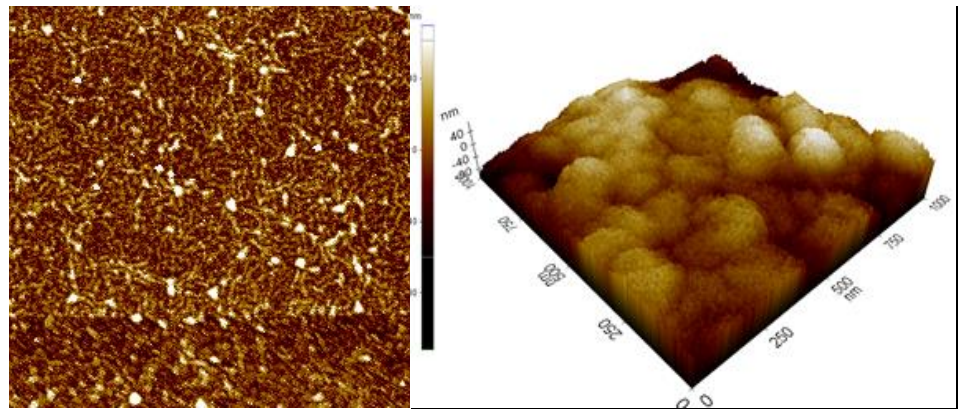

Sekil 3. ㅇ.1 Zn katkılı CuO ince filminin 40umx40um'deki 2 boyutlu ve $1 \mu m \times 1 \mu m$ daki 3 boyutlu görüntüsü.

(Figure 3. AFM image of $\% 0.1 \mathrm{Zn}$ doped CuO thin film for $40 \mu \mathrm{mx} 40 \mu \mathrm{m}, 2$ dimension and in the $1 \mu m \times 1 \mu m$ 3D image)
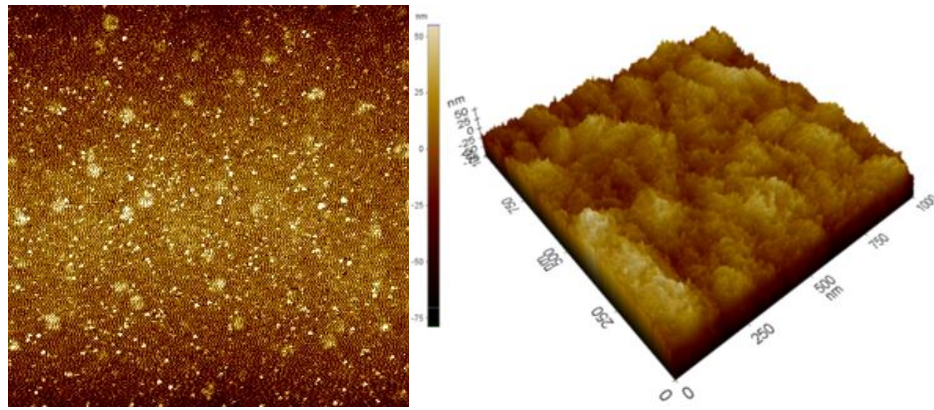

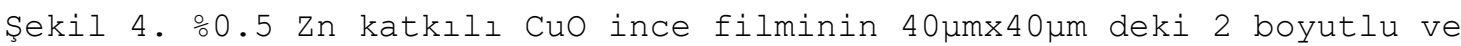
$1 \mu \mathrm{mx} 1 \mu \mathrm{m}$ daki 3 boyutlu görüntüsü.

(Figure 4. AFM image of $00.5 \mathrm{Zn}$ doped CuO thin film for $40 \mu \mathrm{mx} 40 \mu \mathrm{m}, 2$ dimension and in the $1 \mu m \times 1 \mu m$ 3D image)
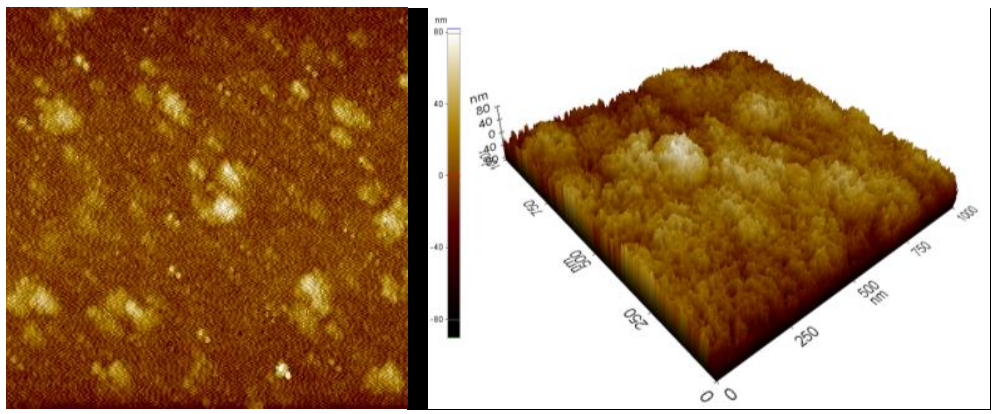

Şekil 5. 2 Zn katkılı CuO ince filminin $40 \mu m x 40 \mu m^{\prime}$ deki 2 boyutlu ve $1 \mu m \times 1 \mu m^{\prime}$ daki 3 boyutlu görüntüsü.

(Figure 5. AFM image of $\frac{0}{2}$. Zn doped CuO thin film for $40 \mu m \times 40 \mu m, 2$ dimension and in the $1 \mu m \times 1 \mu m$ 3D image) 


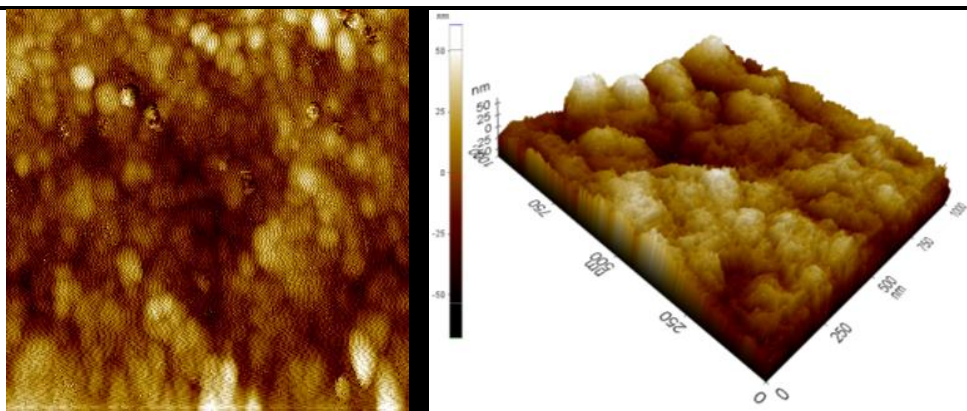

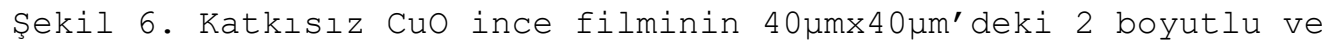
$1 \mu m \times 1 \mu m^{\prime}$ daki 3 boyutlu görüntüsü

(Figure 6. AFM image of undoped CuO thin film for $40 \mu m \times 40 \mu m, 2$ dimension and in the $1 \mu m \times 1 \mu m$ 3D image)

Tablo 1. Katkılı ve Katkısız ince filmlere ait bazı yüzey parametreleri

(Table 1. Some surface parameters doped and undoped for thin films)

\begin{tabular}{|l|c|c|c|}
\hline Numune Adı & $\begin{array}{c}\text { Yüzey Pürüzlülüğü } \\
\left(\mathrm{R}_{\mathrm{q}}\right)(\mathrm{nm})\end{array}$ & $\begin{array}{c}\text { Tane Boyutu } \\
(\mathrm{nm})\end{array}$ & $\begin{array}{c}\text { Eg } \\
(\mathrm{eV})\end{array}$ \\
\hline$\circ 0.1 \mathrm{Zn}$ & 37.33 & $117-169$ & 2.07 \\
\hline$\circ 0.5 \mathrm{Zn}$ & 32.63 & $221-417$ & 1.89 \\
\hline$\circ 2 \mathrm{Zn}$ & 77.89 & $302-917$ & 2.15 \\
\hline Katkısız CuO & 23.96 & $111-208$ & 1.68 \\
\hline
\end{tabular}

\subsection{Al/P-Si/Zn-CuO/Al Diyotlarının I-V Karakteristikleri (I-V Characteristics of $\mathrm{Al} / \mathrm{P}-\mathrm{Si} / \mathrm{Zn}-\mathrm{CuO} / \mathrm{Al}$ Diodes)}

Al/p-Si/Zn:CuO/Al diyotlarının akım voltaj (I-V) karakteristikleri oda sıcaklığında FYTRONIX-FY-5000 güneş pili I-V karakterizasyon sistemi ile analiz edilmiştir. Diyotlar iyi bir doğrultma özelliği gösterdiler. I-V karakteristiklerinin analizi termiyonik emisyon teorisi ile yapıldı. Bu teoriye göre, akım iletim ifadesi;

$$
I=I_{o} \exp \left(\frac{q\left(V-I R_{S}\right)}{n k T}\right)
$$

şeklinde verilir. Burada ( $\left.I_{0}\right)$ ters besleme akımıdır.

$$
I_{o}=A A^{*} T^{2} \exp \left(-\frac{q \Phi_{b}}{k T}\right)
$$

$k$ boltzmann sabiti, $n$ idealite faktörü, $q$ elektron yükü, $T$ mutlak slcaklık, $A^{*}$ etkin Richardson sabiti $\left(32 \mathrm{~A} / \mathrm{cm}^{-2} \mathrm{~K}^{-2}\right.$ for p-tipi Silisyum), A diyotun kontak alanı, ve $\Phi_{b}$ bariyer yüksekliğidir $[30$ ve 31]. 


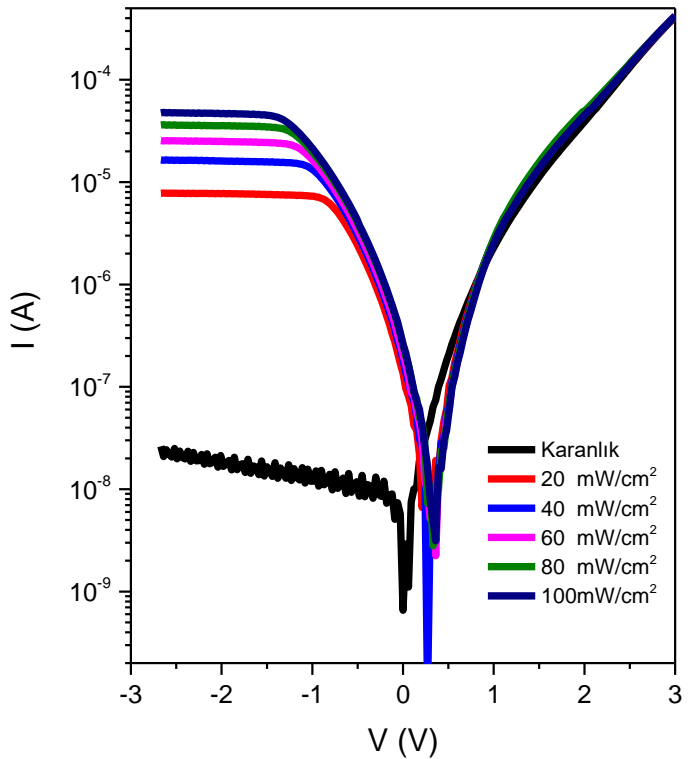

(a) $\div 0.1 \mathrm{Zn}$

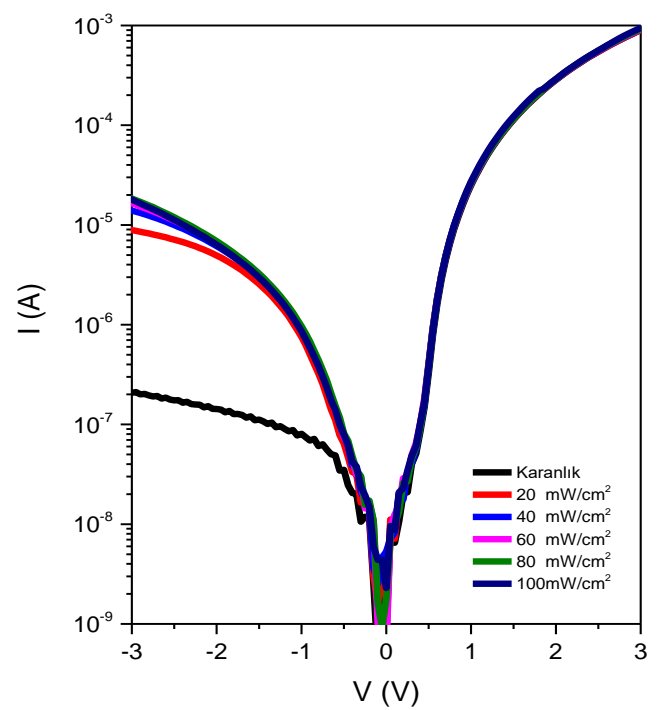

(c) $\div 2$

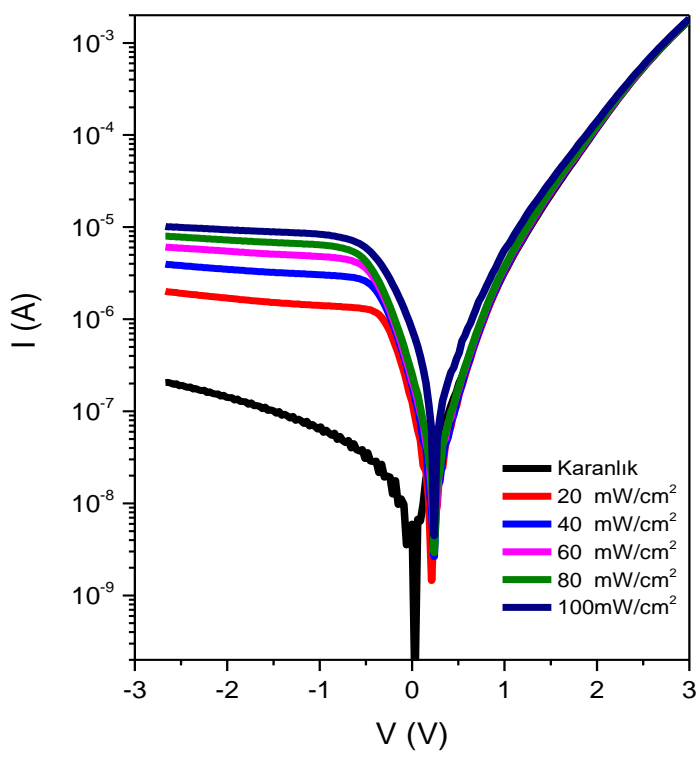

(b) $\div 0.5 \mathrm{Zn}$

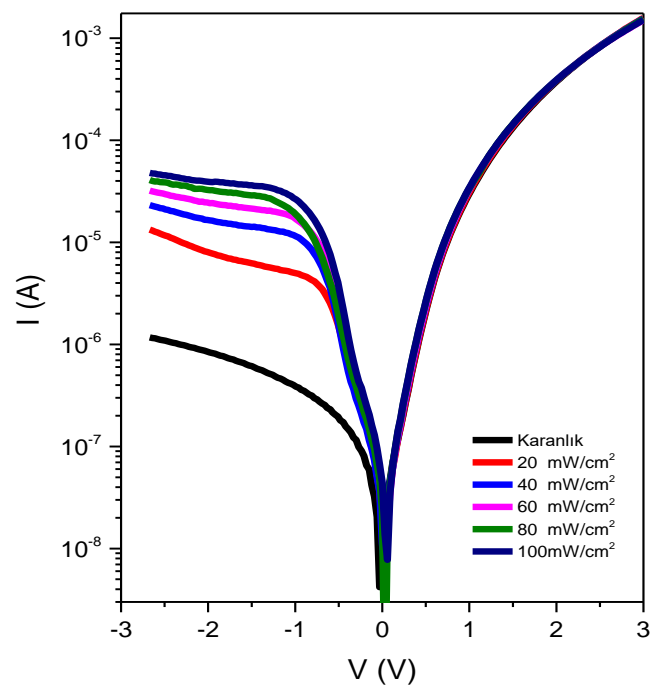

(d) saf $\mathrm{CuO}$

Şekil 4. Al/P-Si/Zn-CuO/Al heteroeklem diyotlarının farklı aydınlatmalardaki akım voltaj (I-V) karakteristikleri

(Fig 4. (a-d) I-V characteristics of Al/P-Si/Zn-CuO/Al heterojuntion diodes at various illumination intensities)

Şekil 4a, b, c ve d görüldüğü gibi diyot akımı, ışık şiddeti ile artmıştır. Işık şiddeti ile elektronlar valans bandından iletim bandına geçerler ve bunun karşılığında diyotun akımı artar. Işıkla fotoakımın artışı, ters besleme bölgesinde, elektron ve boşluk çiftinin oluşmasından kaynaklanır. Diyotların fototepkisi (R) yani belirli bir voltajda fotoakımın, karanlık akıma oranı (fotoakım/karanlık akım) hesaplanmıştır ve en yüksek fototepki ㅇ.1 Zn

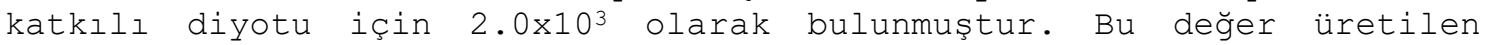




diyotların güneş takip sistemlerinde fotosensör
kullanılabileceğini göstermektedir.

Al/p-Si/Zn-CuO/Al diyotlarının fotoakım-zaman karakteristikleri FYTRONIX-FY-5000 güneş pili I-V karakterizasyon sistemi ile analiz edilmiştir. Diyotların I-t grafikleri Şekil 5 (a-d) de verilmiştir. Şekil 5 te görüldüğü gibi, güneş ışığı altında diyot fotoakımı artmakta ve ışık yok iken ilk değerine geri dönmüştür. Diyotların I-t grafiklerinde görüldüğü gibi, diyotların I açma/ I kapama oranları, o0.1 Zn, 0.5 Zn, 2 Zn ve katkısız CuO için sırasıyla 121.06, 31.36, 27.18, 37.98 olarak bulunmuştur. En yüksek fotoduyarlılığı, \%0.1 Zn katkılı $\mathrm{CuO/p-Si} \mathrm{diyotu} \mathrm{göstermiştir.} \mathrm{Elde} \mathrm{edilen} \mathrm{sonuçlar} \mathrm{diyotların} \mathrm{fotoakım}$ mekanizması, arayüzey bölgesinde oluşan foto taşıyıcılarından kaynaklandığını göstermektedir [32].

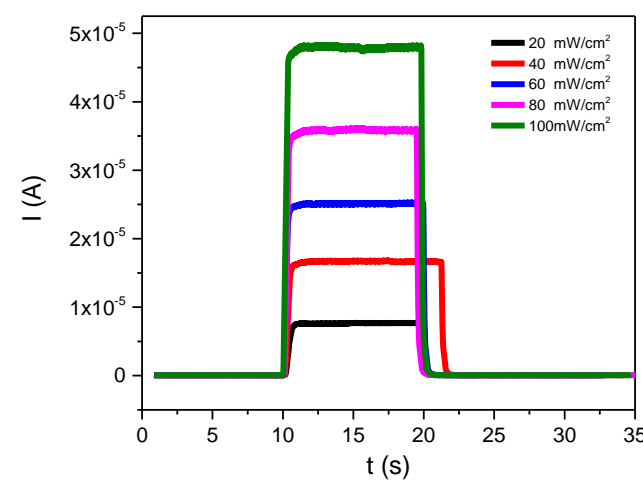

(a) $\div 0.1 \mathrm{Zn}$

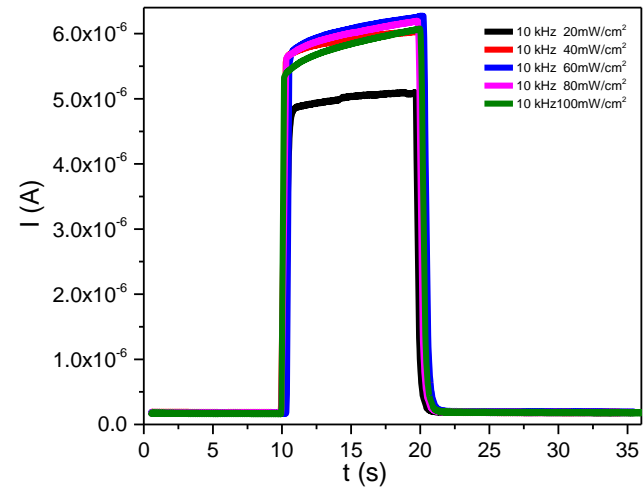

(c) $\div 2 \mathrm{Zn}$

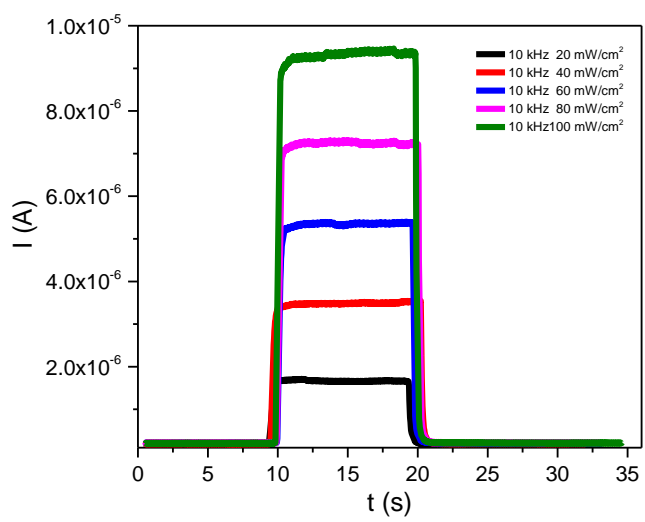

(b) $\div 0.5 \mathrm{Zn}$

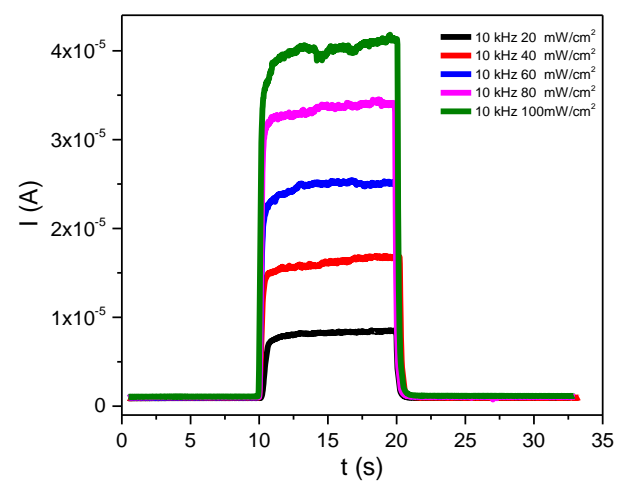

(d) $\mathrm{CuO}$

Şekil 5. Al/p-Si/Zn-CuO/Al heteroeklem diyotlarının akım zaman (I-t) karakteristikleri

(Fig 5. (a-d) Current-time (I-t) characteristics of Al/P-Si/Zn-CuO/Al heterojuntion diodes)

\subsection{Al/p-Si/Zn-CuO/Al Heteroeklem Diyotlarının Kapasite-Voltaj $(C-V)$ Karakteristikleri (Capacitance-Voltage (C-V) Characteristics of $\mathrm{Al} / \mathrm{p}-\mathrm{Si} / \mathrm{Zn}-\mathrm{CuO} / \mathrm{Al}$ Heteroextrusion Diodes)}

$\mathrm{Al} / \mathrm{p}-\mathrm{Si} / \mathrm{Zn}-\mathrm{CuO} / \mathrm{Al}$ heteroeklem diyotlarının kapasite-frekans karakteristikleri FYTRONIX-FY-7000 elektriksel karakterizasyon cihazl ile analiz edilmiştir. Analiz sonuçları Şekil 6 (a-d) verilmiştir. Şekilde görüldüğü gibi frekans artışı ile diyotun kapasitesi azalmıştır. Diyot kapasitesi, pozitif voltaj bölgesinde frekans ile 
değişmezken, negatif voltaj bölgesinde ise frekansla değişmektedir. Negatif bölgede değişim, diyotun tükenim bölgesinin genişliğinin uygulanan negatif voltajla değişiminden kaynaklanmaktadır. C-V eğrilerinde gözlenen pik değeri arayüzey yük taşıyıcılarından ve seri direnç etkisinden kaynaklanmaktadır.

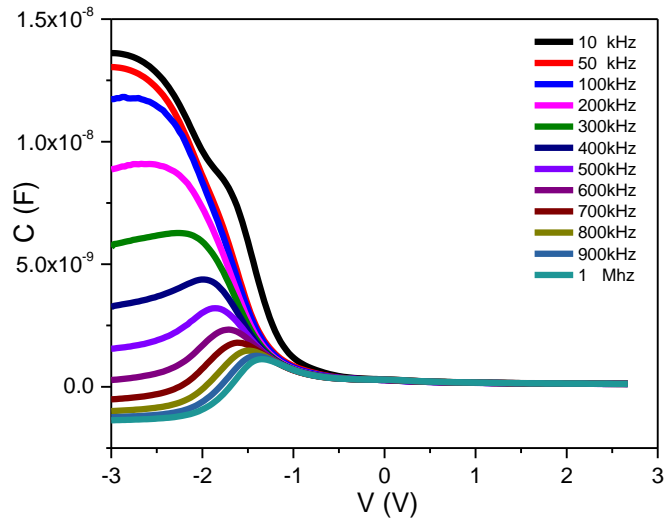

(a) $\div 0.1 \mathrm{Zn}$

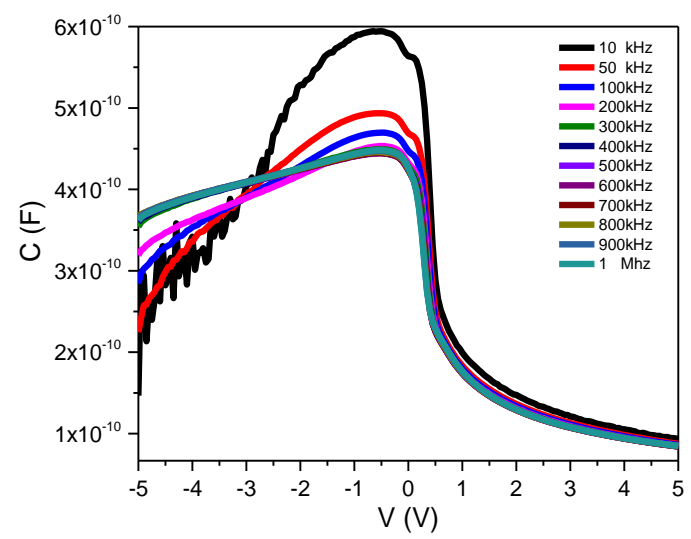

(c) $\div 2 \mathrm{Zn}$

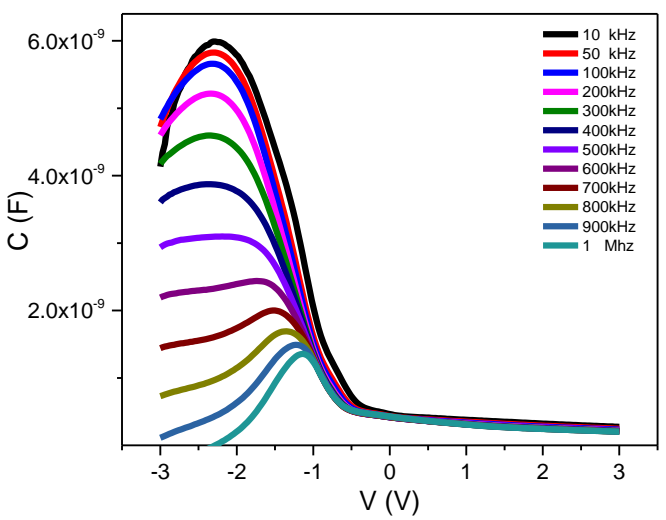

(b) $\div 0.5 \mathrm{Zn}$

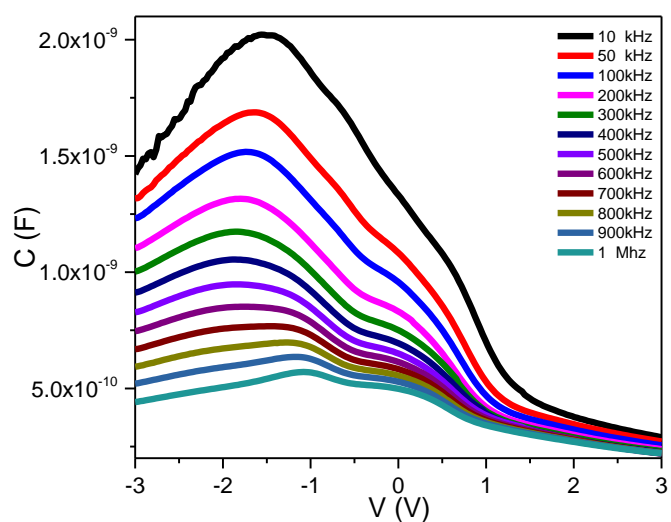

(d) $\mathrm{CuO}$

Şekil 6. Al/p-Si/Zn-CuO/Al heteroeklem diyotlarının kapasite-frekans $(C-V)$ karakteristikleri

(Fig 6. (a-d) Capacity-frequency (C-V) characteristics of Al/P-Si/Zn$\mathrm{CuO/Al}$ heterojuntion diodes)

Şekil 7'de diyotların seri direnç-voltaj eğrileri görülmektedir. Şekilde görüldüğü gibi $R_{s}$ değeri 0.1 ve 0.5 Zn katkılı diyotlarda bir pik gösterirken o2 ve katkısız CuO diyotunda iki pik göstermiştir. Frekans değerinin artmasıyla pik şiddetlerinde azalma görülmüş, bu değişim ara yüzey yüklerinin uygulanan frekansla değişiminden kaynaklanmaktadır. 


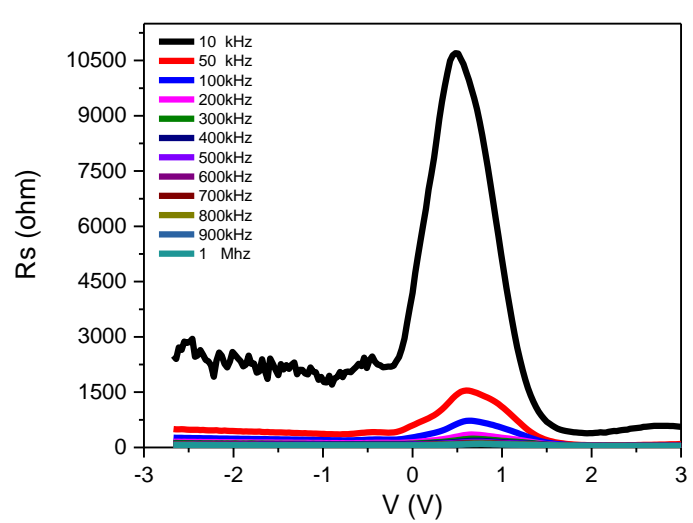

(a) $\div 0.1 \mathrm{Zn}$

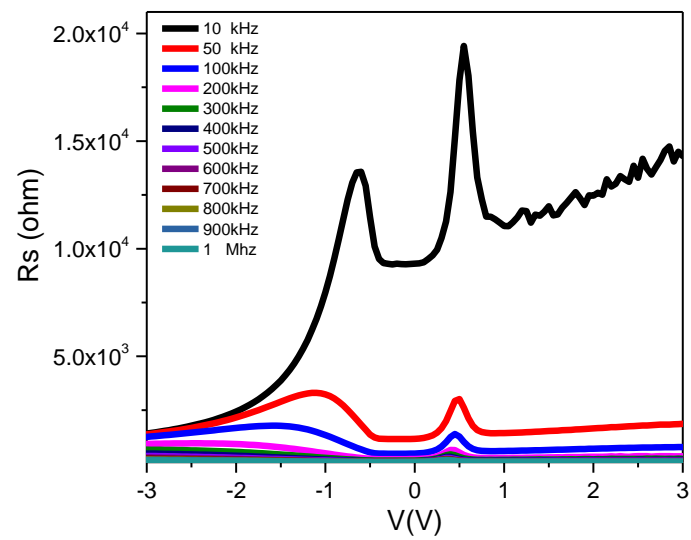

(c) $\div 2 \mathrm{Zn}$

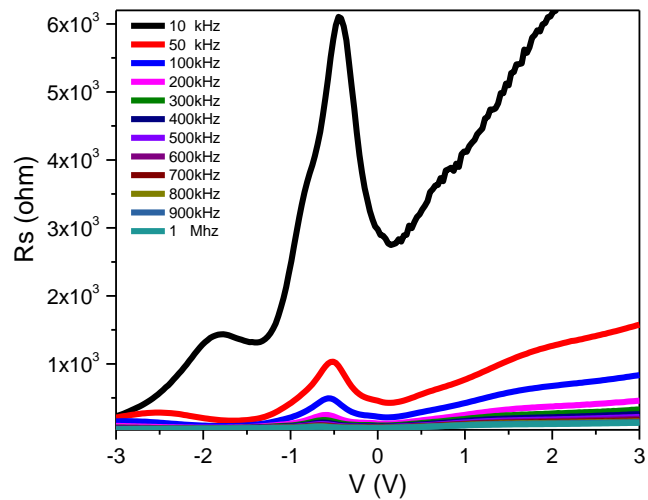

(b) $\div 0.5 \mathrm{Zn}$

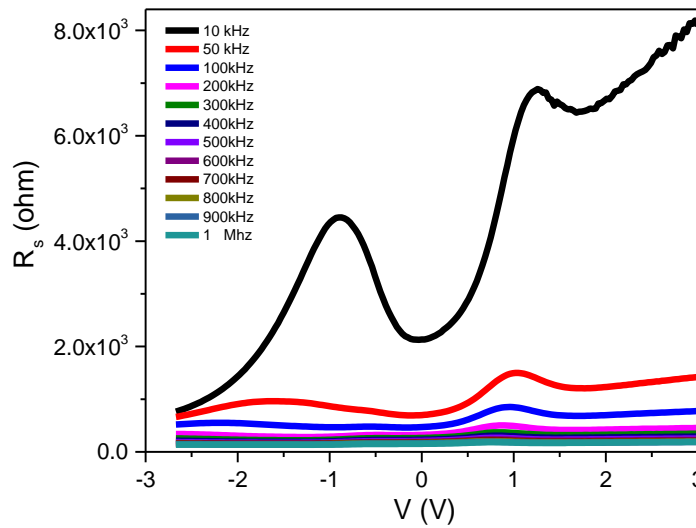

(d) $\mathrm{CuO}$

Şekil 7. Al/p-Si/Zn-CuO/Al heteroeklem diyotlarının seri direnç-voltaj $(R s-V)$ karakteristikleri

(Fig 7. (a-d) Series resistance-voltage $\left(R_{S}-V\right)$ characteristics of Al/P$\mathrm{Si} / \mathrm{Zn}-\mathrm{CuO} / \mathrm{Al}$ heterojuntion diodes)

Şekil 7 (a-d)'den görüldüğü gibi, seri direnç frekans değişimi ile değişmektedir. Bu durum arayüzey yüklerinin uygulanan elektrik alan sinyalini takip ettiğini gösterir.

\section{SONUÇ VE ÖNERILER (CONCLUSION AND RECOMMENDATIONS)}

Zn katkılı CuO heteroeklem diyotları sol-jel spin kaplama metoduyla üretildi. Üretilen diyotların optiksel, yüzeysel ve elektriksel özellikleri araştırıldı. Üretilen diyotlar arasında, en yüksek fototepkiyi ㅇ.1 $\mathrm{Zn}$ katkılı CuO esaslı diyot göstermiştir. Elde edilen sonuçlar, üretilen fotodiyotların güneş takip sistemleri için sensör olarak kullanılabileceğini göstermektedir. 


\section{KAYNAKLAR (REFERENCES)}

[1] Dere, A., (2018). A Novel Quaternary Chalcogenide $\mathrm{KBiCu}_{2} \mathrm{~S}_{3}$ Based Photodiode for Solar Tracking Systems. Physica B: Condensed Matter 545, 30-33.

[2] Wang, S.B., Hsiao, C.H., Chang, S.J., Lam, K.T., Wen, K.H., Hung, S.C., Young, S.J., and Huang, B.R., (2011). A CuO Nanowire Infrared Photodetector. Sensors and Actuators. A 171 207- 211.

[3] Jiang, X.C., Herricks, T., and Xia, Y.N., (2002). CuO Nanowires can be Synthesized by Heating Copper Substrates in Air. Nano Lett. 2, 13331336.

[4] Goodarzi, M.T. and Eshghi, H., (2018). Fabrication and Characterization of CuO/Zno:Al Photo-diode Prepared by Spray Pyrolysis Method. Materials Letters 215, 79-82.

[5] Junying, Z., Li, C., at all., (2009). $\mathrm{Cu}_{2} \mathrm{O}$ thin Films Deposited by Reactive Direct Current Magnetron Sputtering. Thin Solid Films 517, 5700-5704.

[6] Mangamma, G., Jayaraman, V., Gnanasekaran, T., and Periaswami, G., (1998). Effects of SILICA ADDITIONS on HS Sensing Properties of CuO-SnO2 Sensorssens. Actuators B, 53(3), 133-139.

[7] Dandeneau, C.S., Jeon, Yu-H., Shelton, C.T., Plant, T.K., Cann, D.P., Gibbons, B.J., (2009). Thin Film Chemical Sensors Based on p-CuO/n-Zno Heterocontacts Thin Solid Films, 517-15 4448.

[8] Rafea, M.A. and Roushdy, N., (2009). Determination of the Optical Band Gap for Amorphous and Nanocrystalline Copper Oxide Thin Films Prepared by SILAR techniqueJournal of Physics D: Applied Physics, 42 (1) 015413.

[9] Karabat, M.F. ve Arsel, I., (2015). Al/CuO/p-Si/Al Diyot Yapısının Elektriksel Özellikleri. Batman University Journal of Life Sciences; Volume:5 Number: 1.

[10] Gopalakrishna, D., Vijayalakshmi, K., and Ravidhas, C. (2013). Effect of Pyrolytic Temperature on the Properties of NanoStructured CuO Optimized for Ethanol Sensing Applications, J. Mater. Sci.: Mater. Electron. 24, 1004-1011.

[11] Kidowaki, H., Oku, T., Akiyama, T., Jeyadevan, A.S.B., and Cuya, J., (2012). Fabrication and Characterization of CuO-based Solar Cells, J. Mater. Sci. Res. 1, 138-143.

[12] Dere, A., Tataroğlu, A., Al-Sehemi, A.G., Al-Ghamdi, A.A., ElTantawy,F.F., Farooq, W.A., and Yakuphanoglu, F., (2017). A Functional Material Based Photodiode for Solar Tracking Systems. Physica B 520, 76-81.

[13] Steinhauer, S., Brunet, E., Maier, T., Mutinati, G.C., Köck, A., and Freudenberg, O., (2012). Gas Sensing Properties of Novel CuO Nanowire Devices, The 14th International Meeting on Chemical Sensors, 713-716.

[14] Gu, A., Wang G., Zhang X., and Fang B., (2010). Synthesis of CuO Nanoflower and Its Application as a $\mathrm{H}_{2} \mathrm{O}_{2}$ Sensor, Bull. Mater. Sci. 33, 17-20.

[15] Singh, I. and Bedi, R.K., (2011). Studies and Correlation Among the Structural, Electrical and Gas Response Properties of Aerosol Spray Deposited Self-assembled Nanocrystalline CuO, Appl. Surf. Sci. 257, 7592-7599.

[16] Morales, J., Sanchez, L., Martin, F., Ramos-Barrado, J.R., and Sanchez, M., (2004). Nanostructured CuO Thin Film Electrodes Prepared by Spray Pyrolysis: A Simple Method for Enhancing the Electrochemical Performance of CuO in Lithium Cells, Electrochim. Acta 49, 4589-4597.

[17] Gajendiran, J. and Rajendran, V., (2014). Synthesis and Characterization of Coupled Semiconductor Metal Oxide ( $\mathrm{ZnO} / \mathrm{CuO}$ ) Nanocomposite, Mater. Lett. 116, 311-313. 
[18] Shi, R., Yang, P., Zhang, S., and Dong, X., (2014). Growth of Flower-like Zno on Polyhedron CuO Fabricated by a Facile Hydrothermal Method on Cu Substrate, Ceram. Int. 40, 3637-3646.

[19] Chen, K.J., Hung, F.Y., Lui, T.S., Chen, C.H., and Chang, S.P., (2013). The Influences of CuO/Zno Ratios on the Crystallization Characteristics Electrical and Magnetic Properties of Cuxzn1-xO Powders. Hindawi Publishing Corporation Journal of Nanoparticles, Article ID 405043.

[20] Nakamura, Y., Yoshioka, H., Miyayama, M., Yanagida, H., Tsurutani, T., and Nakamura, Y., (1990). Selective CO Gas Sensing Mechanism with CuO/Zno Heterocontact, J. Electrochem. Soc. 137, 940-943.

[21] Saji, K.J., Populoh, S., Tiwari, A.N., and Romanyuk, Y.E., (2013). Design of $\mathrm{p}-\mathrm{CuO} / \mathrm{n}-\mathrm{ZnO}$ Heterojunctions by rf Magnetron Sputtering, Phys. Status Solidi A, 1-6.

[22] Mridha, S. and Basak, D., (2006). Investigation of a p-CuO/n-ZnO Thin Film Heterojunction for H2 Gas-sensor Applications, Semicond. Sci. Technol. 21, 928-932.

[23] Kidowaki, H., Oku, T., and Akiyama, H., (2012). Fabrication and evaluation of $\mathrm{CuO} / \mathrm{ZnO}$ heterojunction for photoelectric conversion, IJRRAS 13, 67-72.

[24] Yang, L., Xie, C., Zhang, G., Zhao, J., Xueli, Y., Zeng, D., and Zhang, S., (2014). Enhanced response to NO2 with CuO/ZnO Laminated Heterojunction Configuration, Sens. Actuat. B 195, 500-508.

[25] Vuong, N.M., Chinh, N.D., Huy, B.T., and Lee, Y., (2016). CuO Decorated Zno Hierarchical Nanostructures as Efficient and Established Sensing Materials for $\mathrm{H}_{2} \mathrm{~S}$ Gas Sensors, Sci. Rep. 6 , 26736.

[26] Yılmaz, M., (2017). İki Eksenli Güneş Takip Sistemlerinde Takip Verimliliğin Arttırılması. Batman University Journal of Life Sciences Volume:7, Number:1/2.

[27] Tauc, J., (1974). Amorphous and Liquid Semiconductors. New York: Plenum Press,

[28] Akkaya, A., (?). The Current-Voltage and Capacitance-Voltage Characterization of Au/Methylene Blue/GaAs Organic-Modified Schottky Diodes.

[29] Dagdelen, F., Serbetci, Z., Gupta, R.K., and Yakuphanoglu, F., (2012). Preparation of Nanostructured Bi-doped CdO Thin Films by Sol-gel Spin Coating Method. Materials Letters 80, 127-130.

[30] Karataş, Ş. and Yakuphanoğlu, F., (2012). Analysis of Electronic Parameters of Nanostructure Copper Doped Cadmium Oxide/p-silicon Heterojunction. Journal of Alloys and Compounds 537, 6-11.

[31] Taşçıoğlu, 亡̇., Tataroğlu, A., Özbay, A., and Altındal, Ş., (2010). The Rolebof 60Co $g$ ray Irradiation on the Interface States and Series Resistance in MIS Structures. Radiation Physicsand Chemistry 79, 457-461.

[32] Özcan, E., Keşan, G., Topaloğlu, B., Tanrıverdi Eçik, E., Dere, A., Yakuphanoğlu, F., and Çosut, B., (2018). Synthesis, Photophysical, DFT and Photodiode Properties of Subphthalocyanine-BODIPY Dyads. New Journal of Chemistry. 1-9. 